\title{
EVAL - An Evaluation Component for Mobile Interfaces
}

\author{
Karin Leichtenstern \\ Institute for Computer Science \\ Eichleitnerstr. 30 \\ 86130 Augsburg, Germany \\ Leichtenstern@informatik. \\ uni-augsburg.de
}

\author{
Dennis Erdmann \\ Institute for Computer Science \\ Eichleitnerstr. 30 \\ 86130 Augsburg, Germany \\ Erdmann@informatik. \\ uni-augsburg.de
}

\author{
Elisabeth André \\ Institute for Computer Science \\ Eichleitnerstr. 30 \\ 86130 Augsburg, Germany \\ Andre@informatik. \\ uni-augsburg.de
}

\begin{abstract}
The Eval Tool is a usability evaluation environment which can be used to evaluate users an their behaviour while they interact with their pervasive computing environment via a mobile phone interface. The tool supports audio-visual recordings of several users and automatically annotates and synchronizes them with context data emerging from the pervasive environment. The annotation of video material with contextual information is important for the analysis of user studies and the detection of usability issues. The EVAL Tool consists of a recorder and a analyzer component. Using the recorder component the capturing of the videos and the logging of the context can be controlled whereas the analyzer components helps to interpret the user study.
\end{abstract}

\section{Categories and Subject Descriptors}

H.5.2 [Information Interfaces and Presentation]: User Interfaces - Evaluation/methodology, Interaction styles, Input devices and strategies, User-centered design

\section{General Terms}

Design, Experimentation, Human Factors

\section{Keywords}

evaluation tool, user study, usability, context

\section{INTRODUCTION}

To develop a mobile phone's application several user studies based on different prototypes are required to finally meet the user's goals. Conducting such a user study is very timeconsuming because videos of the users and their environment have to be captured and interpreted. Thus, a tool would be desirable which supports the following aspects: the audio-visual recording of various users and their environment, the automatic recording of context data and logging of user interactions and the analysis of the captured

(C) Owner/Author | ACM 2008. This is the author's version of the work. It is posted here for your personal use. Not for redistribution. The definitive Version of Record was published in Proceedings of the 10th international conference on Human computer interaction with mobile devices and services - MobileHCI '08, Amsterdam, The Netherlands - September 02 - 05, 2008, S. 483-484

https://doi.org/10.1145/1409240.1409320 data. There have been done less work in the development of evaluations tools. An example for a desktop evaluation tool is DRUM [3]. This tool supports video-assisted usability evaluation. As our evaluation tool DRUM also supports the recording and analysis of videos of a user study. Similar projects as DRUM are UsAGE [4] and KALDI [1] which also support the user studies of desktop applications. The lack of evaluation tools especially the lack of tools to evaluate the mobile phone's applications motivated us to develop the EVAL Tool which helps to record and interpret automatically annotated videos. In the following we introduce this tool and its components.

\section{EVAL TOOL}

The EVAL Tool is a testbed supporting the evaluation of pervasive applications. The tool consists of two components. One component is the EVAL Recorder which supports the capturing of audio-visual user data and the logging of the user's interactions with the mobile phone as well as the context emerging in the pervasive environment. The other component is the EVAL Analyzer which supports an interpretation of the captured data.

\subsection{EVAL Recorder}

The EVAL Recorder can be used to capture users while they are interacting with his pervasive computing environment via a mobile phone. Figure 1 shows the EVAL Recorder's graphical user interface. The tool displays the recorded pictures of two ordinary web cams. To get a comprehensive picture of what is going on while the user is interacting in a pervasive environment, audio-visual recordings are augmented by context data which might be provided by various kinds of sensor, such as a temperature sensor or a GPS sensor. In addition, the EVAL Recorder logs the user's interactions with the GUI of the mobile phone or interactions with the environment, for example, when the user touches an RFID tagged object with the mobile phone. Currently, we log the user's mobile phone input such as the input of commands or text. Moreover, RFID-based and speech-based interactions can also be stored as well as the input of connected input devices such as the Wii. In addition connected sensors such as a temperature sensor or a light sensor can also be logged. In the next version it is planned to detect and log the users' location as well as their activity.

To prevent the logging of irrelevant context, the EVAL Recorder supports the selection of the favored components from a list of all connected users and devices. Another feature of the EVAL Recorder is the display of the incoming 
context from the selected user and the favored components. Moreover, during the whole evaluation process the human evaluator can use the tool to input comments. By connecting more than one EVAL Recorder to the main server, several users can be evaluated. Thus, our testbed even supports the evaluations of multi-user applications for example whenever social context of the different users is an object of the investigation.

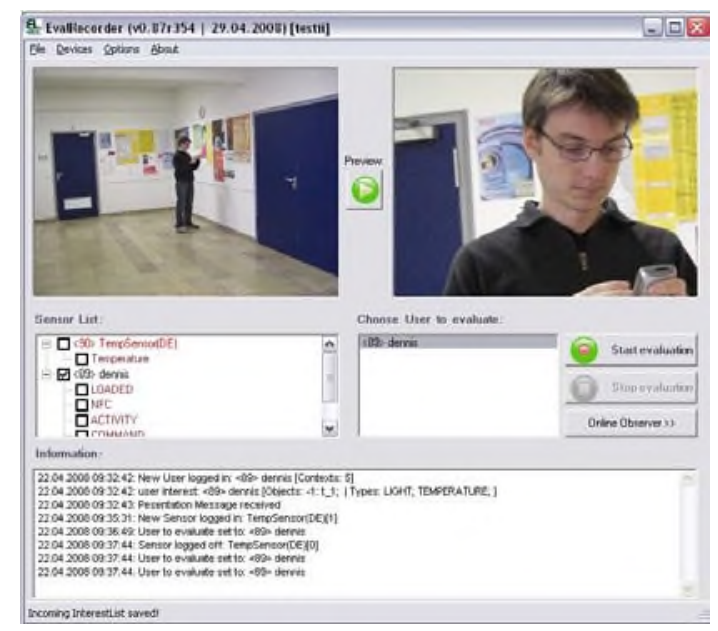

Figure 1: The EVAL Recorder

\subsection{EVAL Analyzer}

After having performed a user study with the EVAL Recorder component, the EVAL Analyzer can be used to interpret the captured data. The Analyser is an extended version of the annotation tool ANVIL [2]. Figure 2 shows the graphical user interface of the EVAL Analyzer. In our example the EVAL Analyzer displays the recorded videos and the corresponding mobile phone's screen. Thus, the evaluator can see which mobile phone screen was displayed at a certain point in time of the interaction. The track panel is created by means of the scheme generated by the EVAL Recorder. In our example, there is a track for the user's interaction and a track for the displayed mobile phone's screen. Within the tracks the corresponding context is displayed. To fill the tracks the stored XML-file from the EVAL Recorder is used. This XML-file contains information about the logged context and its time stamps. Using this information, the evaluator does not need to add this annotation by hand anymore. Instead of, the EVAL Analyzer shows these events which are even the user's interaction with the mobile phone or context within the pervasive environment. The EVAL Analyzer supports the scrolling through the whole captured videos or the switching between different time stamps to observe the user's behavior in a certain context. The evaluator can simply use the tool to interpret the data and to detect potential usability issues. In addition, the EVAL Analyzer enables the addition of further annotations. For example, we may annotate the user's head movements to find out in which situations the user's attention is distracted from the task. Finally, the stored XML-files of the logged context and the manual annotations can also be used for a statistical analysis, for example, to find out which commands lead to user irritation in which context.

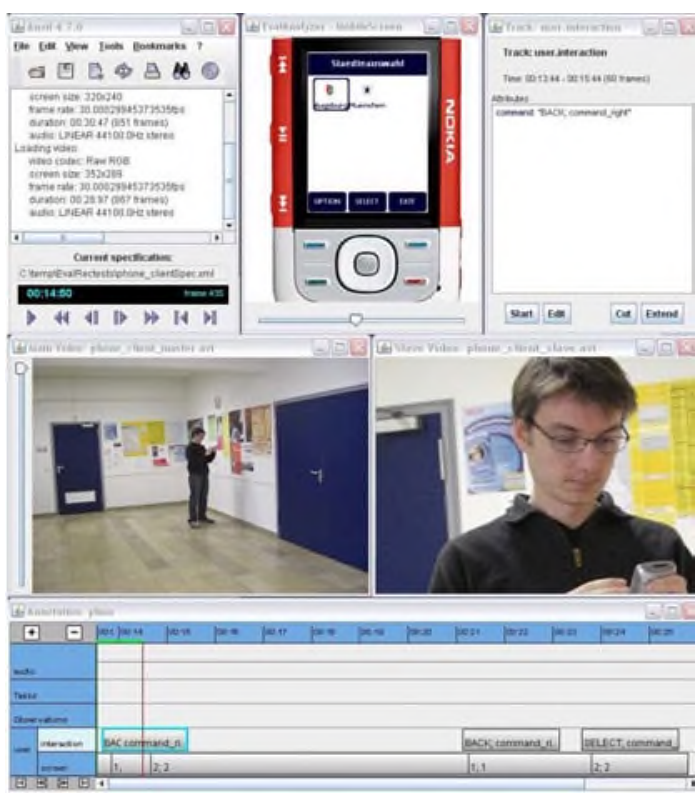

Figure 2: The EVAL Analyzer

\section{CONCLUSION}

In this paper we introduced our evaluation tool for user studies of pervasive applications containing mobile interfaces. The tool called EVAL Tool consists of a recorder and an analyzer component. The recorder supports the recording of two videos and the logging of emerging context in the environment. The analyzer component uses the stored data to automatically annotate the videos by means of the logged context.

\section{ACKNOWLEDGMENTS}

This work was partially supported by European Community (EC) and is currently funded by the eCIRCUS project www.e-circus.org IST-4-027656-STP.

\section{REFERENCES}

[1] G. Al-Qaimari and D. McRostie. Kaldi: a computer-aided usability engineering tool for supporting testing and analysis of human-computer interaction. In Proceedings of the third international conference on Computer-aided design of user interfaces, pages 337-355, Norwell, MA, USA, 1999. Kluwer Academic Publishers.

[2] M. Kipp. Anvil - a generic annotation tool for multimodal dialogue. In Proceedings of the 7th European Conference on Speech Communication and Technology (Eurospeech), pages 1367-1370, Aalborg, September 2001.

[3] M. Macleod and R. Rengger. The development of drum: A software tool for video-assisted usability evaluation. In HCI'93: Proceedings of the HCI conference, pages 293-309, 1993.

[4] D. L. Uehling and K. Wolf. User action graphing effort (usage). In CHI '95: Conference companion on Human factors in computing systems, pages 290-291, New York, NY, USA, 1995. ACM. 